\title{
Design, cloning and expression assay of oipA gene in a bicistronic vector harboring mice IL-18 gene: potential implications for Helicobacter pylori vaccine investigations
}

\author{
Mehran Nemattalab $^{1}$, Mohammad Shenagari ${ }^{1,2 *}$, Ali Mojtahedi ${ }^{1,2}$, Mohammad Reza \\ Aghasadeghi $^{3}$, Mohammad Hassan Pouriayevali ${ }^{3}$, Mojtaba Taheri $^{4}$, Mahdieh Mondanizadeh ${ }^{5}$ \\ 1. Department of Microbiology, Faculty of Medicine, Guilan University of Medical Sciences, \\ Rasht, Iran \\ 2. Cellular and Molecular Research Center, Guilan University of Medical Sciences, Rasht, Iran \\ 3. Department of Hepatitis and AIDS, Pasteur Institute of Iran, Tehran, Iran \\ 4. Department of Biotechnology, Faculty of Para Medicine, Guilan University of Medical \\ Sciences, Rasht, Iran \\ 5. Department of Biotechnology and Molecular Medicine, Arak University of Medical Sciences, \\ Arak, Iran
}

*Corresponding author:Tel: +98 1333690921-229 Fax: +98 1333690036

Address: Department of Microbiology, Faculty of Medicine, Guilan University of Medical Sciences, Rasht, Iran

E-mail: shenagari@gmail.com

Received; 2016/05/24 revised; 2016/07/27 accepted; 2016/08/8

\section{Abstract}

Introduction: Helicobacter pylori $(\mathrm{H}$. pylori) infection has remained as a global health problem. Animal studies demonstrated the role of $\mathrm{H}$. pylori oipA gene in the development of gastric cancer. The aim of this study was the cloning and expression of Helicobacter pylori oipA gene in a bicistronic vector harboring mice IL-18 gene.

Materials and methods: The target gene encoding oipA was amplified from a codonoptimized clone by PCR, and then double-digested by restriction enzymes. The pIRESIgk/mIL18/Fc plasmid was simultaneously digested by BstXI/NotI enzymes to elicit the eGFP segment. PCR product of oipA was inserted into pIRES-Igk/mIL18/Fc plasmid using T4 ligase. Transformation into DH5 $\alpha$ strain was done. Cloning was confirmed by PCR, enzymatic digestion and sequencing. Expression of the oipA and IL-18 mRNA was assessed by means of TaqMan Real-time PCR.

Results: Electrophoresis of PCR product, enzymatic digestion and sequencing showed that the H. pylori oipA gene was successfully cloned into pIRES-Igk/mIL18/Fc to generate mIL18-pIRES2-oipA plasmid. The results of Real-time PCR confirmed the successful expression of both oipA and IL-18 in mouse macrophage cell line.

Conclusion: Considering the role of oipA in pathogenesis of $\mathrm{H}$. pylori and potent activity of IL-18 as a molecular adjuvant, the results of the present study showed that the expression of codon-optimized oipA gene in bicistronic vector including mouse IL-18 is successful. So, it could be considered as an appropriate genetic vaccine candidate for $\mathrm{H}$. pylori in future investigations.

Keywords: Cloning, Codon-optimization, oipA gene, Mouse IL-18, Bicistronic vector

\section{Introduction}

Helicobacter pylori is a Gram negative, motile, spiral-shaped, and microaerophilic microorganism. This bacterium is one of the most important human pathogens that infect more than half of the population worldwide (1). H. pylori is a most important causative agent in some gastroduodenal diseases including duodenal ulcer, peptic ulcer, chronic active gastritis, 
gastric adenocarcinoma, and MALT lymphoma (2).

To date, a number of virulence factors including cagA and vacA genes concerned to severe stomach illnesses such as gastric ulcers and gastric cancer have been identified (3). Almost $4 \%$ of the $\mathrm{H}$. pylori genome encodes outer membrane proteins (OMPs), some of which act as adhesin (4). OipA (Outer inflammatory protein A) is one of the outer membrane proteins which involve in bacterial adhesion. Several studies showed that the oipA gene is another important virulence factor associated with gastric ulcers and cancer (5). Interleukin 18 (IL-18) is considered as a pro-inflammatory cytokine and inducer factor of interferon $\gamma$ (IFN- $\gamma$ ) production by NK cells and CTL (6). Moreover it also increases Fas dependent cell death (FasDependent Cytotoxicity) and Perforin in these cells. On the other hand, this cytokine plays an essential role in $\mathrm{T}$ helper Lymphocyte activities (T helper 1) $(7,8)$. Structurally, IL-18 belongs to the super family of IL-1 and is synthesized in inactive and non- secretory precursor form as same as IL-1 $\beta$ (9). The main characteristic of this cytokine in stimulation of CTL and NK cells and following increased cell killing activity has led to be used in the vaccine research and tumor immunotherapy (10). Therefore, this cytokine can be used as adjuvant to increase vaccine efficiency especially in DNA vaccine protocols (11-13). The importance of OipA in pathogenesis of $\mathrm{H}$. pylori from one side and the adjuvant activity of IL-18 ,especially in TH1 shifting, from other side, persuaded us to design a study to construct a genetic material containing $\mathrm{H}$. pylori oipA and mouse IL-18 genes to assess its immunogenicity in the future investigations.

\section{Material and methods}

In order to clone the target genes, H. pylori oipA and murine IL-18, the pIRES2-eGFP vector (Clontech, Takara Bio Company,
Shiga, Japan) was applied as expression vector. This bicistronic vector allows the expression of two separate protein from the same mRNA by employing IRES (Internal Ribosome Entry Site) element.

As described in previous study, the plasmid containing mouse IL-18 gene fused to murine IgG1 Fc (Fcy2a) and signal sequence of immunoglobulin kappa (IgK) (pIRES-Igk/mIL-18/Fc) was constructed after implementing several cloning and sub-cloning process. As a summary, total RNA of stimulated spleenocytes was extracted and reverse transcribed to produce cDNA of murine IL-18 and Fcy2a. mIL-18/Fc mixed segment was constructed by insertion of Fc fragment and mIL-18 into pSL1180 plasmid. In the following, addition of kappa sign was accomplished by subcloning of $\mathrm{mIL}-18 / \mathrm{Fc}$ into pSectag2a plasmid. Finally, Igk/mIL- $18 / \mathrm{Fc}$ was cloned into pIRES2-eGFP vector using NheI/XmaI enzymes to construct pIRESIgk/mIL-18/Fc plasmid. Identification and confirmation of cloned mIL-18 was assessed by enzymatic digestion, sequencing and ELISA (14).

Codon-optimization of oipA gene: The objective of the present study was cloning of $\mathrm{H}$. pylori oipA gene into previously constructed pIRES-Igk/mIL-18/Fc plasmid. For this purpose, the eGFP coding sequence must be replaced by the desired sequence just after IRES element, using BstXI and NotI restriction enzymes. Regarding to the existence of BstXI restriction site within $\mathrm{H}$. pylori oipA gene, codon optimization strategies was used to eliminate it and improvement of translational efficiency based on Musmusculus codon usage. Modified sequence accuracy was analyzed by NEBcutter V2.0 (New England Biolabs, U.S.A.), SnapGene (GSL Biotech LLC.), BlastX, and Mega 6.0 softwares. The codon-optimized oipA sequence was constructed in BioNeer Co. (Daejeon, Korea).

oipA PCR: The plasmid harboring modified oipA gene was used as template 
for amplification and cloning of this gene into previously constructed pIRESIgk/mIL-18/Fc plasmid. The primers containing restriction sites for BstXI and NotI enzymes were designed using AlleleID v6.0 (PREMIER Biosoft International, USA) and OLIGO 7 (Molecular Biology Insights, Inc., CO, USA) and constructed in BioNeer Co. (Daejeon, Korea) (Table 1). The oipA gene was amplified using Pfu DNA polymerase by PCR with initial denaturation at $94{ }^{\circ} \mathrm{C}$ for $4 \mathrm{~min}$, followed by 25 cycles of denaturation at $94{ }^{\circ} \mathrm{C}$ for $20 \mathrm{~s}$, annealing at $60{ }^{\circ} \mathrm{C}$ for $20 \mathrm{~s}$, elongation at $72{ }^{\circ} \mathrm{C}$ for $2 \mathrm{~min}$ and final extension at 72 ${ }^{\circ} \mathrm{C}$ for $10 \mathrm{~min}$. The final concentrations of reagents in $50 \mu 1 \mathrm{PCR}$ reaction were as follows: $1 \mathrm{X}$ PCR buffer, $2.5 \mathrm{mM} \mathrm{MgCl} 2$, $1 \mathrm{mM}$ dNTP, 50 pmol primers and $15 \mathrm{ng}$ plasmid DNA as template. PCR product was electrophoresed on a $0.8 \%$ low melting agarose gel at $70 \mathrm{~V}$ for $2 \mathrm{~h}$. and purified using QIAquick gel extraction kit (Qiagen, Valencia, CA) according to manufacturer's protocol .

Cloning of oipA gene: Amplified oipA gene and pIRES-Igk/mIL-18/Fc plasmid were double-digested by FastDigest BstXI and fastDigest NotI (Thermo Fisher Scientific, U.S.A). Digested PCR product and plasmid were purified by QIAquick gel extraction kit. Ligation reaction was performed using DNA Ligation kit (Takara Bio Company, Shiga, Japan). The considered molar ratio of vector to insert was 1:3. The recombinant plasmid was transformed into E. coli DH5 $\alpha$ by heatshock protocol and calcium chloride treatment. Finally transformants were selected on LB agar containing $50 \mu \mathrm{g} / \mathrm{ml}$ kanamycin (Sigma-Aldrich, USA) .

Confirmation of Cloned DNA: Colony PCR was conducted using specific primers to evaluate the existence of insert in bacterial colonies. Positive colonies according to PCR results were selected and inoculated into LB broth medium containing $50 \mu \mathrm{g} / \mathrm{ml}$ kanamycin. QIAprep Spin Miniprep kit (Qiagen, Valencia, CA) was utilized for extraction of plasmids. The accuracy of the cloned oipA gene was verified by enzymatic digestion and bidirectional sequencing (BioNeer Company, Korea). The sequence of used primers in colony PCR and bidirectional sequencing were shown in table 1 .

Table 1. List of primer sequences used for cloning of oipA gene in this study.

\begin{tabular}{lr}
\hline & The oipA cloning PCR primers sequences (Amplicon: 954 bp) \\
\hline $\begin{array}{l}\text { oipA F (BstXI) } \\
\text { oipA R (NotI) }\end{array}$ & 5' CTAACCACAACCCGTGGATGAAGAAGGCCCTGTTG 3' \\
\hline & 5' CGGCGCGGCCGCTAATGTTTGTTTTTAAAG 3' \\
\hline oipA-con F & 5' CAACCGTGGATGAAGAAG 3' \\
oipA-con R & 5' GGTATTGAAGCCGTATGG 3' \\
\hline & The sequences of oipA bidirectional sequencing primers \\
\hline oipA-seq F & 5' GCTTTACATGTGTTTAGTCGAGGTT 3', \\
oipA-seq R & 5' CCTCTACAAATGTGGTATGGCTGATT 3'
\end{tabular}

PCR, polymerase chain reaction. $\mathrm{F}$, forward. $\mathrm{R}$, reverse.

Transfection of mIL-18-pIRES2-oipA plasmid: The expression of cloned genes were assessed in eukaryotic cell lines by transfection of constructed mIL-18pIRES2-oipA plasmid into murine macrophage-lineage, RAW 264.7 cell lines. The cells were transfected at $70-90 \%$ confluence using Lipofectamine 2000 (Invitrogen, U.S.A.) with $100 \mathrm{ng}$ of three different plasmids including mIL-18pIRES2-oipA, pIRES-Igk/mIL-18/Fc (As negative control for oipA gene) and pIRES2-eGFP (As positive control of transfection) according to manufacturer's protocol. The efficiency of transfection was analyzed by epifluorescence microscopy. 
Assessment of mIL-18 expression and bioactivity: As previously described by Pouriayevali et al,(14) the expression and bioactivity of cloned mIL-18 from constructed plasmid were evaluated by mouse IL-18 ELISA kit (Bender MedSystems, Austria) and Mouse IFN- $\gamma$ ELISA (U-cytech bioscience, Netherlands) after harvesting the supernatants of transfected RAW 264.7 cell line according to recommendation of manufacturers.

Assessment of oipA expression: To evaluate the expression of cloned oipA gene in transfected cells, TaqMan Realtime PCR technique was used. Total RNA was extracted from transfected RAW 264.7 cells using the RNeasy Mini Kit (Qiagen) and reverse transcription of RNA into cDNA was carried out by HighCapacity cDNA Reverse Transcription Kit (Applied Biosystem, U.S.A.). TaqMan Real-time PCR assay for evaluation of codon-optimized oipA and mIL-18 genes expression was accomplished by designing specific primer and probe using AlleleID software (Table 2). TaqMan Universal PCR Master Mix (Applied Biosystem, U.S.A.) and StepOnePlus Real-time PCR system (Applied Biosystem, U.S.A.) were utilized for running the reaction. The selected method was End-point detection Real-time PCR. The housekeeping mGAPDH (murine glyceraldehyde-3phosphate dehydrogenase) reference gene was considered as Internal Positive Control (IPC). The Real-time PCR reactions were carried out in total volume of $20 \mu \mathrm{l}$ using $10 \mathrm{pmol}$ of specific primer and probes for oipA and mIL-18 separately under the following conditions: $60^{\circ} \mathrm{C}$ for 30 s (Pre-PCR plate read), $50^{\circ} \mathrm{C}$ for $2 \mathrm{~min}$ and $95^{\circ} \mathrm{C}$ for $10 \mathrm{~min}$ (Holding Stage), followed by 45 cycles of $95^{\circ} \mathrm{C}$ for $15 \mathrm{~s}, 60^{\circ} \mathrm{C}$ for $1 \mathrm{~min}$ (Cycling stage) and $60^{\circ} \mathrm{C}$ for 30 s (Post-PCR plate read). mIL18-pIRES2-eGFP and pIRES2-eGFP were used as positive and negative control respectively.

Table 2. The designed primers and probes sequences for Real-time PCR reaction

\begin{tabular}{lc}
\hline mIL-18 -F & 5' AAATGCCCAGCACCTAACCTC 3' \\
mIL-18-R & 5' GGTCATCCTCGCTCACATCC 3' \\
\hline mIL-18-TaqMan & 5' FAM/ACCATCCGTCTTCAT/NFQ-MGB 3' \\
mGAPDH-F & 5' ATGTTCCAGTATGACTCCACTCAC 3' \\
mGAPDH-R & 5' GCTCCTGGAAGATGGTGATGG 3' \\
\hline mGAPDH-TaqMan & 5' 'VIC/ATTCAACGGCACAGTC/NFQ-MGB 3' \\
Mod-oipA-F & 5' GAAGGCTTCTGCACAGAATGC 3' \\
Mod-oipA-R & 5' CGGTTAGCGATTTTGTTTGAATCC 3' \\
Mod-oipA-TaqMan & 5' 'FAM/CCAAGCCATCAACAA/NFQ-MGB 3' \\
\hline
\end{tabular}

PCR, polymerase chain reaction. $\mathrm{F}$, forward. $\mathrm{R}$, reverse.

\section{Results}

Codon-optimization of oipA: The sequencing analysis of ordered plasmid containing modified oipA gene demonstrated the deletion of BstXI restriction site in codon-optimized oipA sequence. Nonetheless, BlastX results confirmed complete homology of codonoptimized and native oipA in coding protein level.

oipA PCR: The electrophoresis of oipA PCR product confirmed the presence of desired $954 \mathrm{bp}$ fragment concerned to this gene (Figure 1).

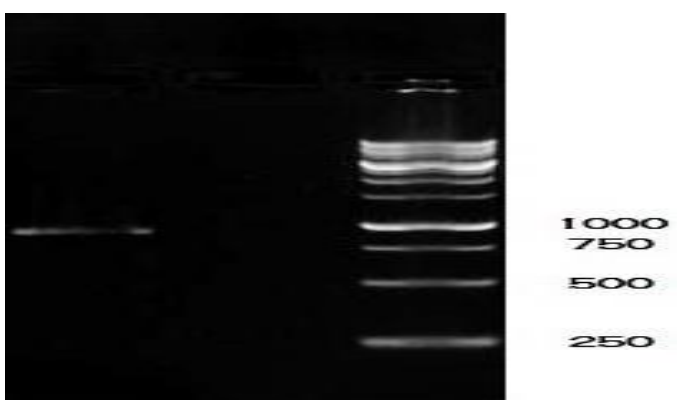

Figure 1. Electrophoresisi of oipA PCR product. 
Confirmation of cloned DNA: The authenticity of cloned DNA was verified by enzymatic digestion, PCR and sequencing. As shown in Figure 2 the inserted 954 bp fragment was brought out after double digestion with BastXI and NotI. Also 459 bp amplicon was produced by plasmid while added as a template in PCR reaction. Analysis of bidirectional sequencing proved complete accuracy of cloned gene.

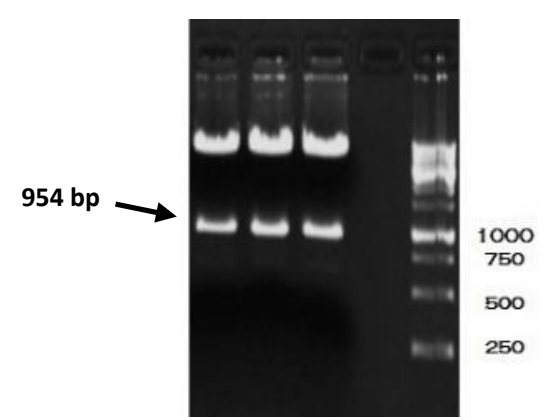

Figure 2. Electrophoresis of confirmatory double digestion.

\section{Recombinant mIL-18 expression and} bioactivity: Assessment of murine IL-18Fcy2a fusion protein expression and biological activity in supernatant of RAW 264.7 cells showed the production of mIL18 near $250 \mathrm{pg} / \mathrm{ml}$ and IFN- $\gamma$ (as a bioactivity indicator) almost $20 \mathrm{pg} / \mathrm{ml}$.

\section{Codon-optimized oipA expression:}

Endpoint TaqMan Real-time PCR assay results showed the successful expression of oipA and mIL-18 genes from mIL-18pIRES2-oipA plasmid in transfected RAW 264.7 cells in comparison to pIRES2eGFP (as negative control).

\section{Discussion}

Despite great progress in $\mathrm{H}$. pylori vaccine design, the mechanisms of mucosal immunity against this bacterium is unclear up to now. In fact, it is surprising that proteins such as urease and heat shock protein homologues which are present in the cytoplasm of other intestinal pathogens may be serves as vaccines against $H$. pylori. Successful H. pylori vaccination strategies were evaluated in different animal models by oral administration of $\mathrm{H}$. pylori antigens in combination with mucosal adjuvants such as cholera toxin and E. coli LT enterotoxin. Various antigens have been used as prophylactic or therapeutic $H$. pylori vaccines in animal models. Chen et al in 2012 reported that injection of the recombinant OipA protein in combination with IL-2 molecular adjuvant and pLTB genes could be effective against $\mathrm{H}$. pylori by reducing the bacterial load in immunized mice (16). In another study, Chen and colleagues showed that injection of genetic vaccine expressing oipA loaded on attenuated Salmonella typhimurium strain in C57 mice can effectively stimulate cellular and humoral immunity (18). Falivene et al in 2012 showed the importance of IL-18 cytokine in effective immunization strategies against antigens carried by MVA vector (Modified Vaccinia Ankara) by deleting IL-18 binding protein gene in this vector (17). Due to the ability of IL-18 in stimulation of innate and adaptive immune responses and Th1 shifting, DNA vaccine containing IL-18 molecular adjuvant sounds appealing strategies in preventing of classic and re-emerging diseases (19). Several advantages have been cited for fusion of murine Fcy2a to cytokines including increased avidity, prolonged circulating half-life due to molecular size increase and reduced renal clearance. Furthermore, fusion with $\mathrm{Fc}$ results in alteration of complement-mediated and antibody dependent cell-mediated cytotoxicity, enhancing the effects of IL18 as a molecular adjuvant (14). 
Considering OipA virulence and carcinogenesis, the aim of present study was to build a bicistronic plasmid containing codon-optimized $\mathrm{H}$. pylori oipA and murine IL-18 genes to assess immunogenicity of this plasmid in animal models in future studies. Selection of a bicistronic vector enabled us to express both oipA and IL-18 genes from the same construct. Applying two plasmids in DNA vaccine projects can hamper the reproducibility of experiments, so bicistronic vector was used to ensure equal molar ratio of genes (Equimolar) in DNA vaccine formulation in ongoing study. The

\section{References}

1. Mojtahedi A, Salehi R, Navabakbar F, Tamizifar H, Tavakkoli H, Duronio V. Evaluation of apoptosis induction using PARP cleavage on gastric adenocarcinoma and fibroblast cell lines by different strains of Helicobacter pylori. Pak J Biol Sci. 2007; 10(22):4097-102.

2. Ghasemian Safaei H, Tavakkoli H, Mojtahedi A, Salehei R, Soleimani B, Pishva E. Correlation of cagA positive Helicobacter pylori Infection with clinical outcomes in Alzahra hospital, Isfahan, Iran. J Res Med Sci. 2008; 13(4):196-201.

3. Kusters JG, van Vliet AH, Kuipers EJ. Pathogenesis of Helicobacter pylori infection. Clin Microbiol Rev. 2006; 19(3):449-90.

4. Yamaoka Y. Increasing evidence of the role of Helicobacter pylori SabA in the pathogenesis of gastroduodenal disease. J Infect Dev Ctries. 2008; 2(3):174-81.

5. Yamaoka Y, Kwon DH, Graham DY. A M(r) 34,000 proinflammatory outer membrane protein (oipA) of results of sequencing, transfection and RTPCR showed that H. pylori optimized oipA gene as well as murine IL-18 were expressed successfully from constructed mIL-18-pIRES2-oipA plasmid in monocytes / macrophages cell line. Moreover, the application of this construct is possible as a promising $\mathrm{H}$. pylori DNA vaccine candidate.

\section{Acknowledgments}

We thank N. Hajipour, K. Asadpour and M. Ahmadi for valuable helps. This research was supported by grant from Guilan University of Medical sciences.

Helicobacter pylori. Proc Natl Acad Sci USA. 2000; 97(13):7533-8.

6. Ushio S, Namba M, Okura T, Hattori K, Nukada Y, Akita K, Tanabe F, et al. Cloning of the cDNA for human IFNgamma-inducing factor, expression in Escherichia coli, and studies on the biologic activities of the protein. J Immunol. 1996; 156(11):4274-9.

7. Netea MG1, Kullberg BJ, Verschueren I, Van Der Meer JW. Interleukin-18 induces production of proinflammatory cytokines in mice: no intermediate role for the cytokines of the tumor necrosis factor family and interleukin-1beta. Eur J Immunol. 2000; 30(10): 3057-60.

8. Micallef MJ, Ohtsuki T, Kohno K, Tanabe F, Ushio S, Namba M, et al. Interferon-gamma-inducing factor enhances $\mathrm{T}$ helper 1 cytokine production by stimulated human $\mathrm{T}$ cells: synergism with interleukin-12 for interferon-gamma production. Eur J Immunol.1996; 26(7):1647-51.

9. Dinarello CA. Interleukin-18. Methods. 1999; 19(1): 121-32.

10. Barouch DH1, Santra S, Tenner-Racz K, Racz P, Kuroda MJ, Schmitz JE, et 
al. Potent CD4+ $\mathrm{T}$ cell responses elicited by a bicistronic HIV-1 DNA vaccine expressing gp120 and GMCSF. J Immunol. 2002; 168(2): 562-8.

11. Seki E, Tsutsui H, Nakano $H$, Tsuji $\mathrm{N}$, Hoshino K, Adachi O, et al. Lipopolysaccharide-induced IL-18 secretion from murine Kupffer cells independently of myeloid differentiation factor 88 that is critically involved in induction of production of IL-12 and IL-1beta. J Immunol. 2001; 166(4): 2651-7.

12. Abdulhaqq SA, Weiner DB. DNA vaccines: developing new strategies to enhance immune responses. Immunol Res; 2008; 42(1-3): 219-32.

13. Yamaoka Y, Kikuchi S, el-Zimaity HM, Gutierrez O, Osato MS, Graham DY. Importance of Helicobacter pylori oipA in clinical presentation, gastric inflammation, and mucosal interleukin 8 production. Gastroenterology. 2002; 123(2): 414-24.

14. Chi CH, Lin CY, Sheu BS, Yang $\mathrm{HB}$, Huang AH, Wu JJ. Quadruple therapy containing amoxicillin and tetracycline is an effective regimen to rescue failed triple therapy by overcoming the antimicrobial resistance of Helicobacter pylori. Aliment Pharmacol Ther, 2003; 18(3): 347-53.

15. Pouriayevali, $\mathrm{MH}$, Memarnejadian AR, Sadat SM, Zavvar M, Siadat SD,
Hartoonian C, et al. Designing and construction of bicistronic plasmid pIRES-Igk/mIL18/Fc: potential implications for vaccine investigations. Pathobiology. 2011; 14(2): 13-23.

16. Chen J, Lin L, Li N, She F. Enhancement of Helicobacter pylori outer inflammatory protein DNA vaccine efficacy by co-delivery of interleukin-2 and B subunit heat-labile toxin gene encoded plasmids. Microbiol Immunol. 2012; 56(2): 8592.

17. Falivene J, Del Médico Zajac MP, Pascutti MF, Rodríguez AM, Maeto C, Perdiguero B, et al. Improving the MVA vaccine potential by deleting the viral gene coding for the IL-18 binding protein. PLoS One. 2012; 7(2): e32220.

18. Chen J, Li N, She F. Helicobacter pylori outer inflammatory protein DNA vaccine-loaded bacterial ghost enhances immune protective efficacy in C57BL/6 mice. Vaccine. 2014; 32(46): 6054-60.

19. Wan YY, Flavell RA. How diverse-CD4 effector $\mathrm{T}$ cells and their functions. J Mol Cell Biol. 2009; 1(1):20-36.

20. Vazquez-Lombardi R, Roome B and Christ D. Molecular engineering of therapeutic cytokines. Antibodies. 2013; 2; 426-451. 